\title{
Recent advances in blood flow vector velocity imaging
}

Jensen, Jørgen Arendt; Nikolov, Svetoslav; Udesen, Jesper; Munk, Peter; Hansen, Kristoffer Lindskov; Pedersen, Mads Møller; Hansen, Peter Møller; Nielsen, Michael Bachmann; Oddershede, Niels; Kortbek, Jacob

Total number of authors:

12

Published in:

2011 IEEE International Ultrasonics Symposium

Link to article, DOI:

10.1109/ULTSYM.2011.0064

Publication date:

2011

Document Version

Early version, also known as pre-print

Link back to DTU Orbit

Citation (APA):

Jensen, J. A., Nikolov, S., Udesen, J., Munk, P., Hansen, K. L., Pedersen, M. M., Hansen, P. M., Nielsen, M. B., Oddershede, N., Kortbek, J., Pihl, M. J., \& Li, Y. (2011). Recent advances in blood flow vector velocity imaging. In 2011 IEEE International Ultrasonics Symposium (pp. 262 - 271). IEEE.

https://doi.org/10.1109/ULTSYM.2011.0064

\section{General rights}

Copyright and moral rights for the publications made accessible in the public portal are retained by the authors and/or other copyright owners and it is a condition of accessing publications that users recognise and abide by the legal requirements associated with these rights.

- Users may download and print one copy of any publication from the public portal for the purpose of private study or research.

- You may not further distribute the material or use it for any profit-making activity or commercial gain

- You may freely distribute the URL identifying the publication in the public portal 
Paper presented at the IEEE International Ultrasonics Symposium, Orlando Florida, 2011:

\section{Recent advances in blood flow vector velocity imaging}

Jørgen Arendt Jensen ${ }^{1}$, Svetoslav Ivanov Nikolov ${ }^{2}$, Jesper Udesen ${ }^{4}$, Peter Munk ${ }^{1}$ Kristoffer Lindskov Hansen ${ }^{3}$, Mads Mфller Pedersen ${ }^{3}$, Peter Møller Hansen ${ }^{3}$, Michael Bachmann Nielsen ${ }^{3}$, Niels Oddershede ${ }^{4}$, Jacob Kortbek ${ }^{2}$, Michael Johannes Pihl ${ }^{1}$ and $\mathrm{Ye} \mathrm{Li}^{1}$

${ }^{1}$ Center for Fast Ultrasound Imaging, Biomedical Engineering group, Department of Electrical Engineering, Bldg. 349, Technical University of Denmark, DK-2800 Kgs. Lyngby, Denmark

${ }^{2}$ BK Medical, Mileparken 34, DK-2730 Herlev, Denmark

${ }^{3}$ Department of Radiology, Copenhagen University Hospital, Rigshospitalet, Denmark ${ }^{4}$ GN ReSound A/S, Lautrupbjerg 9, DK-2750 Ballerup, Denmark.

To be published in Proceedings of IEEE International Ultrasonics Symposium, Orlando Florida, 2011. 


\title{
Recent advances in blood flow vector velocity imaging
}

\author{
Jørgen Arendt Jensen ${ }^{1}$, Svetoslav Ivanov Nikolov ${ }^{2}$, Jesper Udesen ${ }^{4}$, Peter Munk ${ }^{1}$ \\ Kristoffer Lindskov Hansen ${ }^{3}$, Mads Møller Pedersen ${ }^{3}$, Peter Møller Hansen ${ }^{3}$, Michael Bachmann Nielsen ${ }^{3}$, \\ Niels Oddershede ${ }^{4}$, Jacob Kortbek ${ }^{2}$, Michael Johannes Pihl ${ }^{1}$ and ${\mathrm{Ye} \mathrm{Li}^{1}}^{1}$ \\ ${ }^{1}$ Center for Fast Ultrasound Imaging, Dept. of Elec. Eng. Bldg. 349, \\ Technical University of Denmark, DK-2800 Lyngby, Denmark \\ ${ }^{2}$ BK Medical, Mileparken 34, DK-2730 Herlev, Denmark \\ ${ }^{3}$ Department of Radiology, Copenhagen University Hospital, Rigshospitalet, Denmark \\ ${ }^{4}$ GN ReSound A/S, Lautrupbjerg 9, DK-2750 Ballerup, Denmark.
}

\begin{abstract}
A number of methods for ultrasound vector velocity imaging are presented in the paper. The transverse oscillation (TO) method can estimate the velocity transverse to the ultrasound beam by introducing a lateral oscillation in the received ultrasound field. The approach has been thoroughly investigated using both simulations, flow rig measurements, and in-vivo validation against MR scans. The TO method obtains a relative accuracy of $10 \%$ for a fully transverse flow in both simulations and flow rig experiments. In-vivo studies performed on 11 healthy volunteers comparing the TO method with magnetic resonance phase contrast angiography (MRA) revealed a correlation between the stroke volume estimated by TO and MRA of $0.91(p<0.01)$ with an equation for the line of regression given as: $\mathrm{MRA}=1.1 \cdot$ TO-0.4 $\mathrm{ml}$. Several clinical examples of complex flow in e.g. bifurcations and around valves have been acquired using a commercial implementation of the method (BK Medical ProFocus Ultraview scanner). A range of other methods are also presented. This includes synthetic aperture imaging using either spherical or plane waves with velocity estimation performed with directional beamforming or speckle tracking. The key advantages of these techniques are very fast imaging that can attain an order of magnitude higher precision than conventional methods. SA flow imaging was implemented on the experimental scanner RASMUS using an 8-emission spherical emission sequence and reception of 64 channels on a BK Medical 8804 transducer. This resulted in a relative standard deviation of $1.2 \%$ for a fully transverse flow. Plane wave imaging was also implemented on the RASMUS scanner and a $100 \mathrm{~Hz}$ frame rate was attained. Several vector velocity image sequences of complex flow were acquired, which demonstrates the benefits of fast vector flow imaging. A method for extending the 2D TO method to $3 D$ vector velocity estimation is presented and the implications for future vector velocity imaging is indicated.
\end{abstract}

\section{Velocity Estimation AND its Limitations}

Medical ultrasound has for more than 40 years been capable of estimating the blood velocity in the human cardiovascular system. This is of major diagnostic value for screening and investigating haemodynamic problems. Current commercial systems, however, only estimate the velocity along the ultrasound direction, which often is the least significant velocity component as the major vessels run parallel to the skin [1], [2], [3]. Also at bifurcations, valves, and geometric changes the flow is not uni-directional and varies as a function of space and phase in the cardiac cycle.

All of the current systems find the axial velocity along the ultrasound direction and, thus, give the projected velocity component. In spectral systems this is compensated for by visually determining the angle and dividing by the $\cos (\theta)$-factor to yield quantitative velocities. The underlying assumption is that there is one static direction, which can be compensated for. This presupposes that the flow is laminar along the vessel walls. Clinical measurements by several authors [4], [5] have revealed that this is a gross simplification for most clinical situations. No fully laminar flow is found in a complex, pulsating flow system with short, curved and branching vessels. Often vortices are found in parts of the cardiac cycle and helical and secondary flow are found in most curving and branching arteries. The motion will, thus, in general not follow the vessel boundaries, and the direction varies over the cardiac cycle and as a function of spatial position. Static angle compensation methods can therefore not be used in a quantitative velocity estimation system.

For spectral systems angle errors can result in very erroneous estimates even in straight vessels with non-perturbed flow [6]. At constrictions e.g. atherosclerosis or at bifurcations entirely wrong velocity estimates can occur for spectral systems. Only at part of the cardiac cycle for e.g. a valve will the resulting spectrum be correct. In color flow mapping the images are very difficult, if not impossible, to interpret for the complex flow encountered in the human circulation. Changes in angle can result in dramatic changes in the appearance of the image with sudden changes in color and often unrealistically low velocities are show as seen in Fig. 1. The image shows the carotid bifurcation for a healthy volunteer right after peak systole. The velocity here is around $0.8-1 \mathrm{~m} / \mathrm{s}$, which is not shown due to the angle problem. Also the image contains a mixture of colors due to the various velocity directions that change over spatial position and time. A standard method for improving the angle between the flow and the ultrasound direction is to angle the beams, when the vessel wall and transducer surface are parallel. The example shown in Fig. 1 clearly demonstrates that this will not alleviate the problem. 


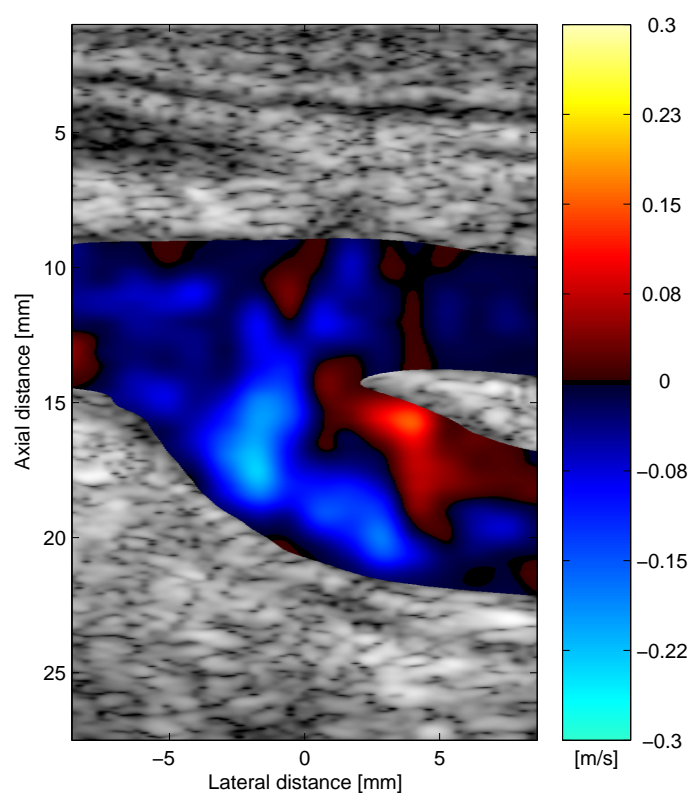

Fig. 1. CFM images of the carotid bifurcation for a healthy volunteer right after peak systole.

There is, thus, a real need for velocity estimation systems that can find the velocity vector in the scan plane. Several authors have suggested methods for measuring the velocity vector. Fox [7] devised an approach for using two intersecting beams to deduce both the axial and the transverse component. The angle between the beams should be sufficient to ensure a reliable transverse estimate and this limits the accuracy and depth for scanning. Trahey et al [8] suggested using speckle tracking where two (small) images are correlated to find the translation in two directions. This requires a rapid image acquisition to get sufficient data for the tracking. Another method using speckle tracking is echo particle image velocimetry where a contrast agent is injected into the blood stream. Images of the particles are here correlated to find the vector velocity [9], [10]. The major drawback is here of course the use of a contrast agent.

Newhouse et al. [11] suggested using the bandwidth of the transverse beam as a measure of the transverse velocity, but this is influenced by many factors including the axial velocity [12]. A method for determining the flow angle was suggested by Tortoli et al. [13], where a spectral range gate is placed with a beam direction at right angles to the vessel, and the direction adjusted so that the spectrum is always symmetric around zero frequency. A second beam is then used for finding the spectrum. The approach has to assume that the angle is fixed, which is not the case in the human body. A review of the early history of vector Doppler has been published by Dunmire and Beach [14] and a more recent review is given by Evans and Jensen [15].

This paper will present various methods for vector velocity estimation developed by the authors. The transverse oscillation method is presented in Section II, which also gives several examples from its use both in flow rigs and in the clinic. The method is fairly efficient to implement as it only demands a parallel beamformer and four times the amount of calculations as for a traditional autocorrelation estimation. The method has the usual limitations in frame rate based on aliasing limits and sequential data acquisitions. Such limitations can be alleviated by using more advanced imaging schemes like synthetic aperture imaging and plane wave imaging. These are the topics of Sections III and IV. For both these techniques very fast imaging can be attained and significantly more accurate velocity estimates can be obtained, since data are available for the whole image simultaneously. The paper is concluded with a description of further directions for velocity estimation and what this might lead to of measurement systems and clinical possibilities.

\section{Transverse OSCILlation Vector Flow IMAgING}

This section describes the transverse oscillation (TO) approach, reveal its performance, and show clinical examples of its use from both experimental and commercial ultrasound scanners.

In conventional velocity estimation, ultrasound pulses with a center frequency of $f_{0}$ are emitted repeatedly in one direction every $T_{p r f}$ seconds. The returned signal $r(t, i)$ is sampled at the depth of interest $D$ determined by the time $t_{z}$ since pulse emission. This yields a signal for a single moving scatterer given by [2]:

$$
\begin{aligned}
r\left(t_{z}, i\right) & =a \sin \left(2 \pi f_{0}\left(t_{z}-\frac{2 D}{c}-i t_{s}\right)\right) \\
& =a \sin \left(2 \pi f_{0}\left(t_{z}-\frac{2 D}{c}-\frac{2 v_{z}}{c} i T_{p r f}\right)\right)
\end{aligned}
$$

where $c$ is the speed of sound, $v_{z}$ is the scatterer velocity, and $a$ the scattering amplitude. The time shift of the scatterers between pulse emissions is:

$$
t_{s}=\frac{2 v_{z}}{c} T_{p r f}
$$

The received signal is a function of $i$ the pulse emission number and the resulting sampled signals is

$$
r_{s}(i)=-a \sin \left(2 \pi \frac{2 v_{z}}{c} f_{0} i T_{p r f}-\phi_{d}\right)
$$

where $\phi_{d}$ is the arbitrary phase factor for this depth. The received frequency is, thus, given by

$$
f_{p}=\frac{2 v_{z}}{c} f_{0}=\frac{2|\vec{v}| \cos \theta}{c}
$$

and is directly proportional to the blood velocity, where $\theta$ is the flow to ultrasound beam angle. The key aspect in this is the regular sampling of the sinusoidal wave, where the time shift $t_{s}$ between emissions give rise to a received sinusoidal wave with a frequency of $f_{p}$.

\section{A. Transverse Oscillation Method}

There is no harmonic oscillation transverse to the ultrasound beam and the received signal is, thus, not influenced by a transverse motion. The TO approach [16], [17] introduces a 

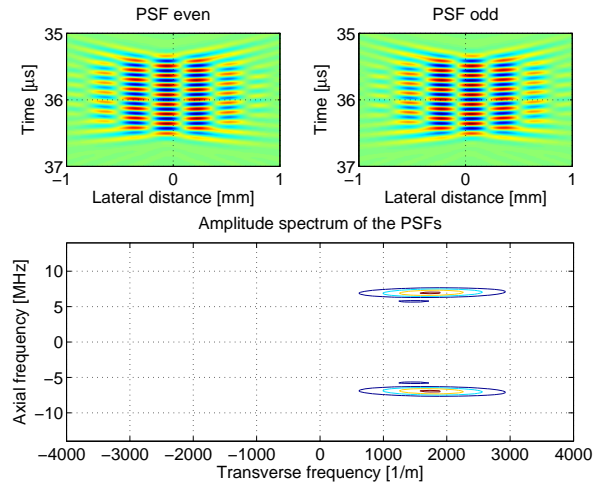

Fig. 2. The two Field II simulated double oscillating point spread functions (PSFs) (top) and the corresponding spectrum (bottom) for a point at 27 $\mathrm{mm}$ using a linear-array transducer with a center frequency of $7 \mathrm{MHz}$. The amplitude spectrum is calculated by taking the two-dimensional Fourier transform of PSFeven $+j$ PSFodd. Note that the PSFs are $90^{\circ}$ phase shifted with respect to each other and that the amplitude spectrum, therefore, is onesided (from [20]).

lateral oscillation in the ultrasound field to make it influenced by the transverse velocity.

The transverse oscillation is introduced by emitting a normal focused ultrasound field and then receiving on two parts of the transducer aperture simultaneously to generate a sinusoidal oscillation. In the far field there is a Fourier relation between the transducer aperture function and the pressure field [16]. An aperture function containing two peaks spaced a distance $l$ will, thus, yield a sinusoidal oscillation given by:

$$
r_{t}(i)=\cos \left(2 \pi f_{p} i T_{p r f}+\phi\right) \cos \left(2 \pi \frac{v_{x}}{\lambda_{x}} i T_{p r f}\right)
$$

where $v_{x}$ is the transverse velocity and $\lambda_{x}$ is the lateral modulation period. The lateral extend of the field is determined by the width and shape of the two peaks on the aperture. An example of such a field is shown in Fig. 2. They were simulated for a linear array transducer with the Field II program [18], [19].

The lateral oscillation period $\lambda_{x}$ is determined by the distance between the two peaks $d$ and the depth $z_{0}$ by:

$$
\lambda_{x}=\frac{2 \lambda_{z} z_{0}}{d}
$$

where $\lambda_{z}$ is the axial wavelength. The field can be created by a traditional focused transmission and then the lateral oscillation is created during the receive beamforming. This also has the advantage that the distance between the peaks can be dynamically adjusted as a function of depth to maintain a constant lateral oscillation [21].

The sign of the lateral velocity should also be determined. This is found from having a complex signal created during the receive beamforming by having a $90^{\circ}$ phase shift between two parallel receive beams. They are spaced $\lambda_{x} / 4$ apart to yield a spatial quadrature signal. A temporal Hilbert transform is performed on both beams to generate four received signal from which two estimators can give the axial and lateral velocity independently and hence the 2D velocity vector [22].

The received and sampled spatial quadrature signal is

$$
r_{s q}(i)=\cos \left(2 \pi f_{p} i T_{p r f}\right) \exp \left(j 2 \pi f_{x} i T_{p r f}\right) .
$$

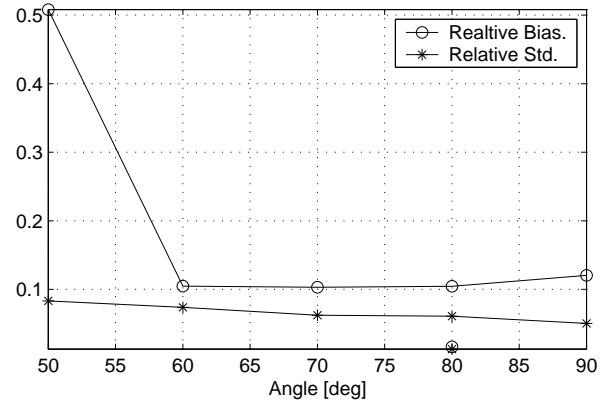

Fig. 3. Relative bias and standard deviation for the TO method when measuring parabolic flow in a straight tube (from [20]).

The temporal Hilbert transform of the received signal is

$$
r_{s q h}(i)=\sin \left(2 \pi f_{p} i T_{p r f}\right) \exp \left(j 2 \pi f_{x} i T_{p r f}\right) .
$$

Two new signals are then formed from:

$$
\begin{aligned}
& r_{1}(i)=r_{s q}(i)+j r_{s q h}(i) \\
& r_{2}(i)=r_{s q}(i)-j r_{s q h}(i)
\end{aligned}
$$

The estimators are then [22]:

$$
v_{x}=\frac{\lambda_{x}}{2 \pi 2 T_{p r f}} \arctan \left(\frac{\mathfrak{I}\left\{R_{1}(1)\right\} \mathfrak{R}\left\{R_{2}(1)\right\}+\mathfrak{I}\left\{R_{2}(1)\right\} \mathfrak{R}\left\{R_{1}(1)\right\}}{\mathfrak{R}\left\{R_{1}(1)\right\} \mathfrak{R}\left\{R_{2}(1)\right\}-\mathfrak{I}\left\{R_{1}(1)\right\} \mathfrak{I}\left\{R_{2}(1)\right\}}\right)
$$

and

$$
v_{z}=\frac{\lambda_{z}}{2 \pi 4 T_{p r f}} \arctan \left(\frac{\mathfrak{I}\left\{R_{1}(1)\right\} \mathfrak{R}\left\{R_{2}(1)\right\}-\mathfrak{I}\left\{R_{2}(1)\right\} \mathfrak{R}\left\{R_{1}(1)\right\}}{\mathfrak{R}\left\{R_{1}(1)\right\} \mathfrak{R}\left\{R_{2}(1)\right\}+\mathfrak{I}\left\{R_{1}(1)\right\} \mathfrak{I}\left\{R_{2}(1)\right\}},\right.
$$

where $R_{1}(1)$ is the complex lag one autocorrelation value for $r_{1}(i)$ and $R_{2}(1)$ is the complex lag one autocorrelation value for $r_{2}(i)$. $\mathfrak{I}$ denotes imaginary part and $\mathfrak{R}$ real part.

The axial and transverse velocity components are then estimated independently hereby yielding the velocity vector. The advantage of the TO method is its fairly low amount of calculations and the dual or triple beam-former employed by the methods already exits in most high end scanners of today.

\section{B. Performance}

The performance of the TO approach was investigated using a circulating flow rig and the experimental RASMUS scanner [23]. A $7 \mathrm{MHz}$ linear array probe with 128 elements was employed and 32 emissions were used for each estimate. The flow rig had a fully developed parabolic velocity profile and the relative bias and standard deviation was calculated and are shown in Fig. 3. The standard deviation is on the order of 5\% and the bias is around $10 \%$ apart from at $50^{\circ}$ flow angle where problems are found with reverberations in the flow rig.

\section{Clinical Examples and Validation}

The TO approach was initially implemented on the experimental RASMUS scanner [23] for revealing the clinical possibilities and performance. An example from the carotid bifurcation from a healthy male is shown in Fig. 4. The left graph shows the flow vectors before peak systole and the right shows after the peak. The color intensity and vector lengths indicate velocity magnitudes and the vectors also 

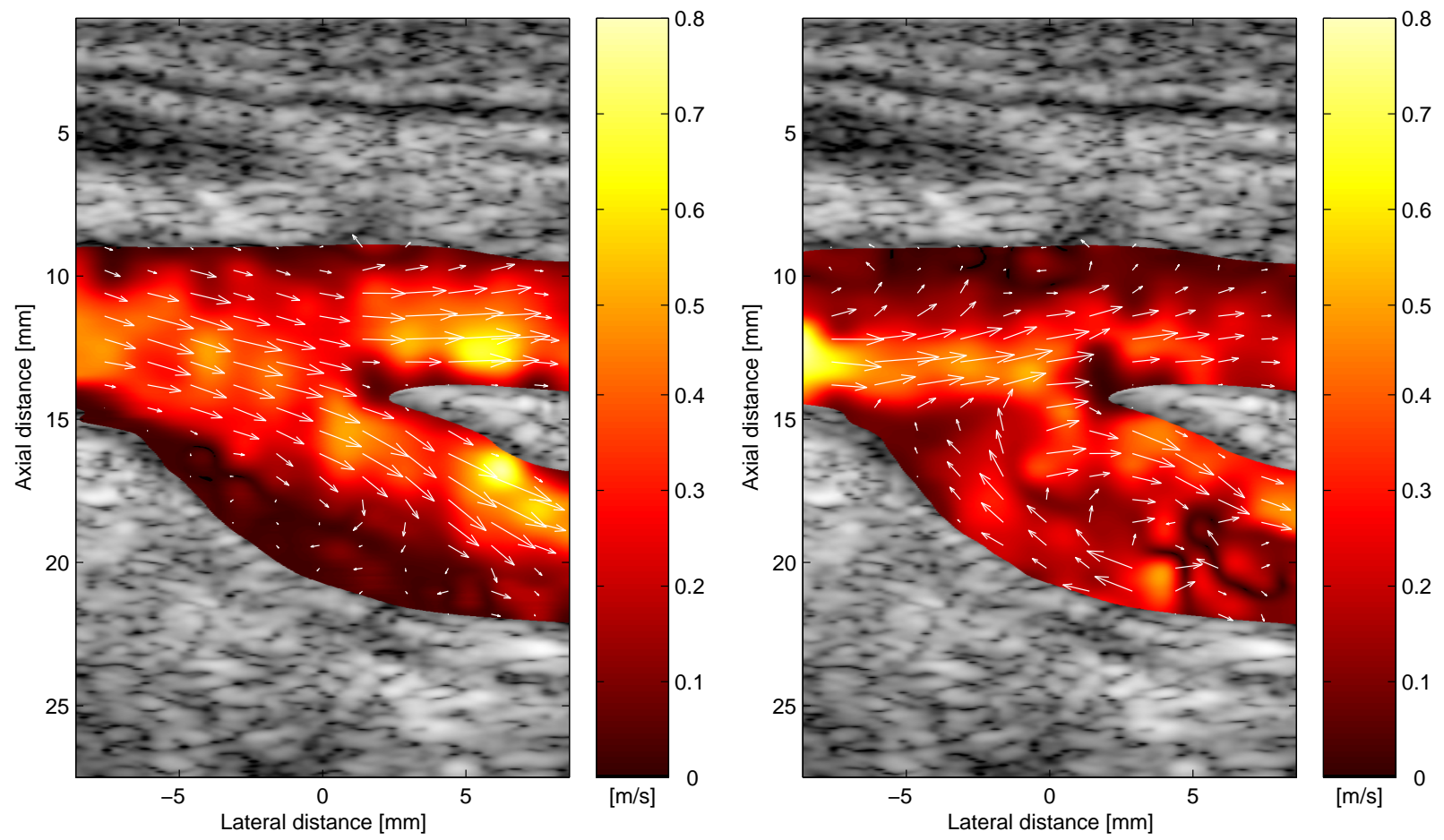

Fig. 4. Vector velocity image of the carotid artery bifurcation shortly before (left) and after (right) peak systole (from [24]). The arrows indicates both direction and magnitude of the velocity and color intensity indicates velocity magnitude.

indicate direction. The example demonstrates how the velocity direction vary rapidly (within $50 \mathrm{~ms}$ ) and completely changes direction depending on both the spatial location and time in the cardiac cycle. The flow before peak systole is fairly laminar, whereas the vortex flow after peak systole have components in all directions. Also the vortex travels down stream at approximately $0.5 \mathrm{~m} / \mathrm{s}$ and the flow again approaches a laminar flow. Note also how much of the flow is perpendicular to the ultrasound beam, and, thus, cannot be measured by a conventional system. The acquired data can also be used to find the traditional axial velocity component and this is shown in Fig. 1 for comparison. Note here how the velocities are often an order of magnitude lower than in the vector flow image, and that there is not a fixed angle that can compensate for this. This will be the case for any complex, pulsating flow for e.g. bifurcations and branches, around stenoses, and at all types of valves.

A validation of the TO approach against MR phased contrast velocity imaging has been conducted by Hansen et al. [25] Eleven volunteers had their carotid artery scanned with both TO ultrasound and MR angiography. The angle between the ultrasound beam and the flow was $90^{\circ}$. From these data the stroke volume was calculated and compared as shown in Fig. 5. The correlation between the stroke volume estimated by TO and MRA was $0.91(p<0.01)$ with the equation for the line of regression: $\mathrm{MRA}=1.1 \cdot \mathrm{TO}-0.4 \mathrm{ml}$. It should be noted that the scans were not performed on the same day as the scanners were placed at different locations.

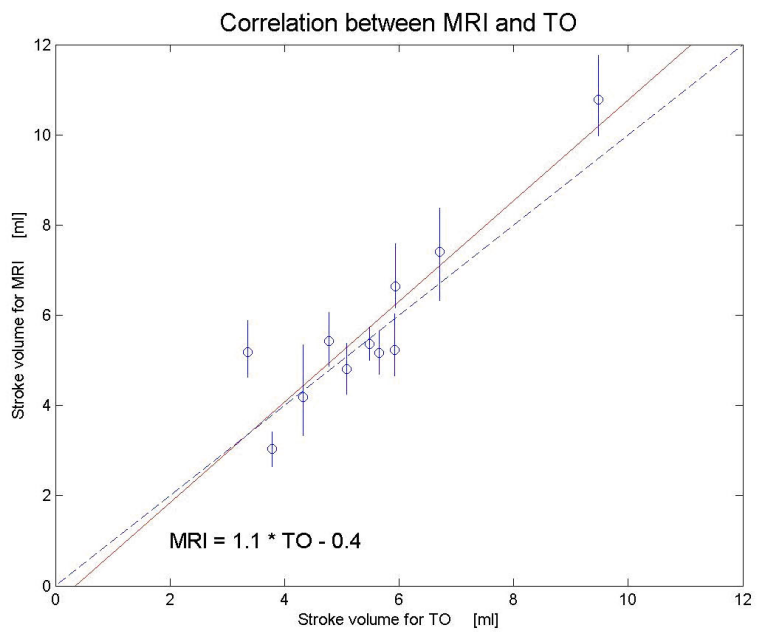

Fig. 5. Correlation between measurements of the stroke volume in the carotid artery using the TO approach and a phased contrast MR scan (from [25]).

\section{Implementation}

The TO method has been implemented on a BK Medical UltraView scanner (BK Medical, Herlev, Denmark) and can now display real-time vector velocity images at 20-30 frames per second using a linear array transducer. The display of velocity magnitude and direction is shown in Fig. 6, where the magnitude is encoded as color intensity and direction is shown both by the vector and the color.

The first clinical example shows secondary flow in the crossectional plane of the abdominal aorta. At parts of the 


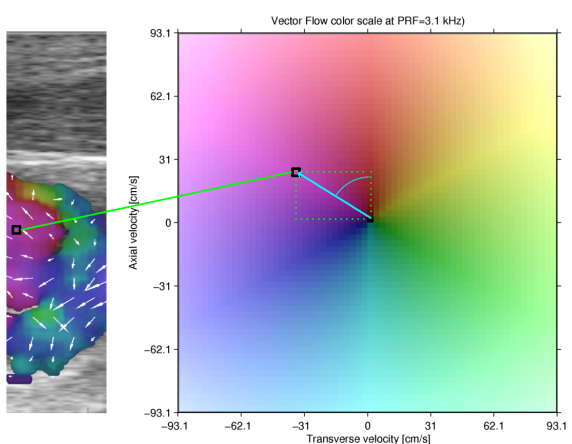

Fig. 6. The velocity vectors are encoded as both arrows and in a color scale, where the brightness indicates magnitude and the color direction.

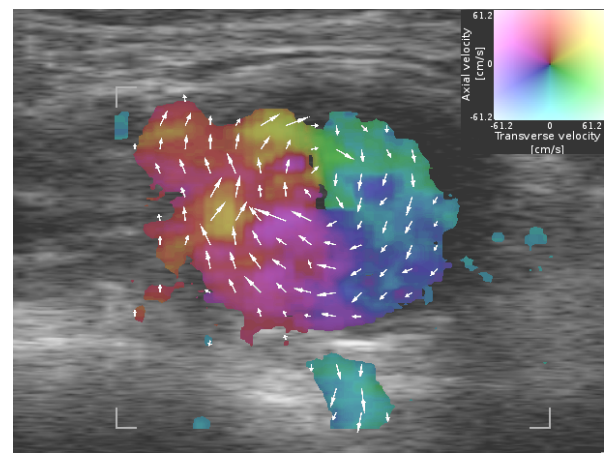

Fig. 7. Secondary flow in the abdominal aorta (modified figure from [26]).

cardiac cycle a rotation of the flow is found and can now be visualized. Note how all flow directions are present in the image, and how the full color scale is used. the vectors point in all directions and nearly the full color scale is used.

The second example in Fig. 8 shows the complex velocity patterns obtained around a valve in the femoral vein. The valve closure gives rise to a vortex behind the leaflet and flow in many directions is again seen.

\section{Synthetic Aperture Vector Flow Imaging}

Synthetic aperture imaging is a radical break with traditional sequential ultrasound imaging. Here images are not acquired one line at a time and the transmitted field is often not focused. The approach has several challenges in terms of penetration, motion artifacts, and the number of calculations. This Section

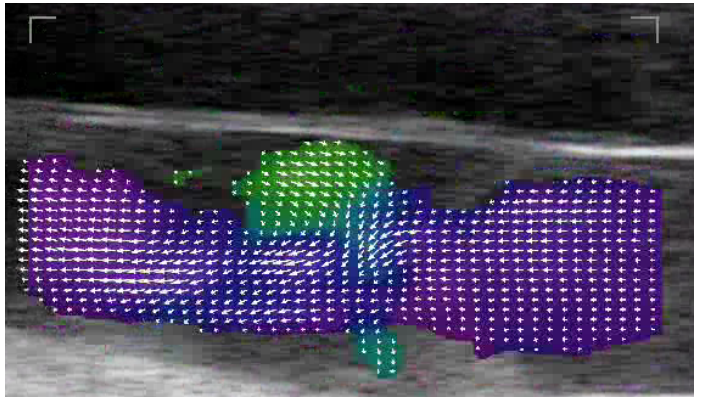

Fig. 8. Longitudinal scan of the femoral vein with disturbed flow at the passage of a venous valve. A vortex is formed in the pocket behind the valve (modified figure from [26]).

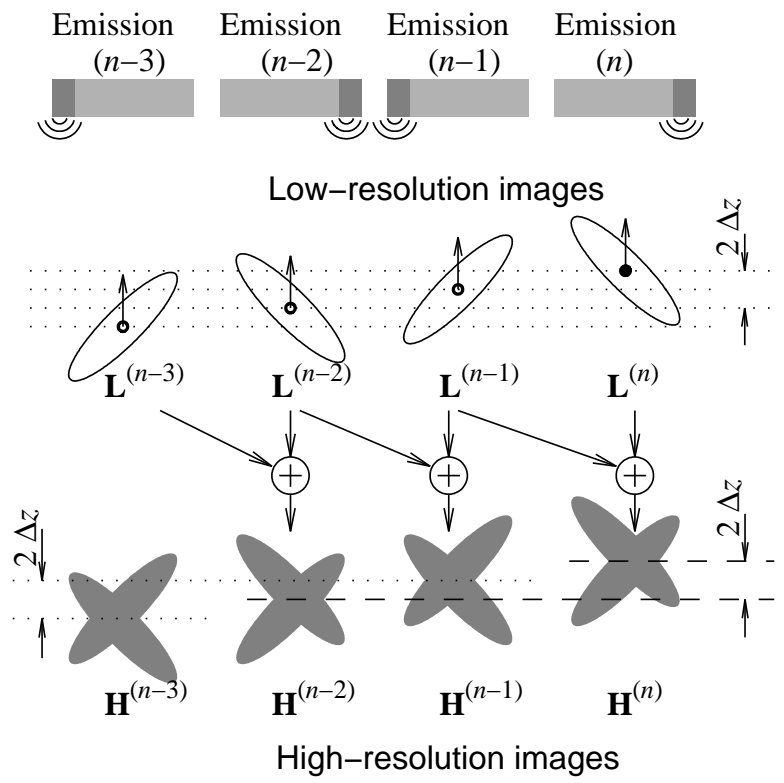

Fig. 9. Emission sequence from synthetic aperture flow imaging (from [30]).

will show that these can be overcome and the approach can be used for very precise vector velocity estimation.

\section{A. Method}

In synthetic aperture imaging a spherical transmission is used to insonify the whole image region. The scattered signal is received by all transducer elements. A low resolution image can then be made with focusing in receive only. The process is repeated over the whole aperture and all low resolution images are added in phase. This make the resulting images dynamically focused in both transmit and receive and creates the best possible delay-and-sum beamformed image [27]. Also the method can maintain a high frame rate, if only few emissions are used.

There are several challenges in SA imaging for clinical imaging. Firstly, the spherical transmission lowers the emitted energy and this must be compensated for. Gammelmark and Jensen [28] showed that this could be overcome by combining several elements using spherical focus delays and chirp excitation to emit more energy and increase the penetration depth compared to traditional imaging. The approach was also shown to give superior images in a double blinded comparison to traditional imaging [29].

The challenge has then been to adapt this to flow imaging, as the image is reconstructed from several pulse emissions and the scatterers, thus, move during the acquisition. This results in a blurred image. The solution is to use short emission sequences as shown in Fig. 9. Here a sequence using only two emissions are repeated. Combining the first and second emission gives a point spread function that is the same for every emission pair apart from the axial translation.

It is, thus, possible to correlate the signals for every set of $N$ emissions, where $N$ is the length of the emission sequence. This is shown in Fig. 10. 


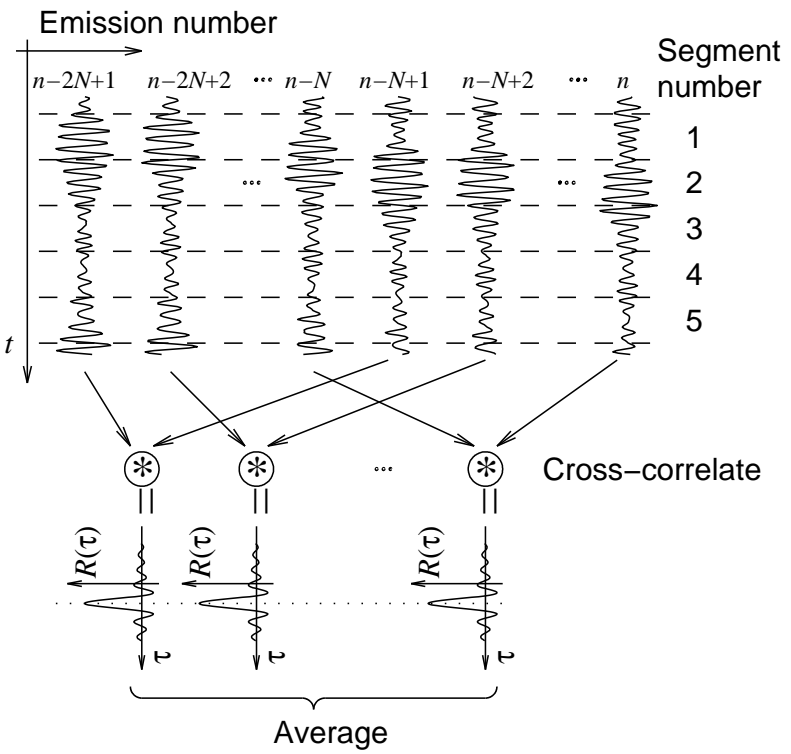

Fig. 10. Cross-correlation of continuous high resolution data for finding the velocity (from [30]).

There are several advantages to this compared to conventional flow imaging. The image acquisition is continuous in time over the full image. It is therefore possible to have continuous signals from everywhere in the image. The velocity estimate can, thus, be averaged over as long time as the velocity can be considered stationary and is not limited by the number of emissions in one direction. This significantly increases velocity accuracy and makes it possible to use any kind of stationary echo canceling filter without initialization effects.

The first example of an in-vivo SA image is shown in Fig. 11. An emission sequence with 4 emission spaced over the 128 elements linear array was used and this was repeated 6 times. The full color flow image of the axial velocity was then estimated from these 24 emissions. In this scheme it is possible to display more than 200 CFM images a second for $f_{\text {prf }}=5 \mathrm{kHz}$ corresponding to scanning down to a depth of $15 \mathrm{~cm}$. More emissions can also be used to average the results to gain a more accurate response or to visualize slow moving flow.

An advantage of SA imaging is that a full data set is acquired and there is no limitation on how the beamformation can be performed. It is possible to beamform in any direction and along any path in the image. This has been used to devise a scheme where received signals are beamformed along the flow direction as shown in Fig. 12.

Short lines along the beam to flow angle are beamformed centered around the position where the velocity should be determined. The lines are then cross-correlated to find the shift in position along the line and divided by the time between emissions directly gives the velocity magnitude. The approach has been shown to work for both traditional imaging [32], [33] and for synthetic aperture flow imaging [31]. A key issue is determining the angle for the flow. This can be attained by

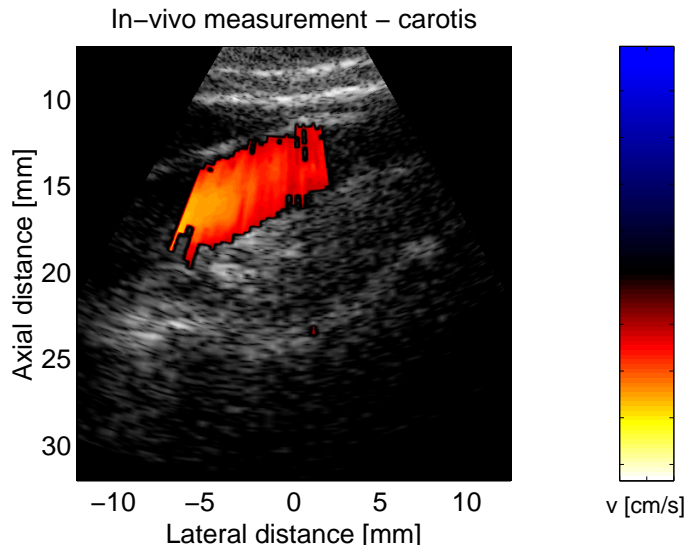

Fig. 11. In-vivo SA flow image from the carotid artery (from [30]).

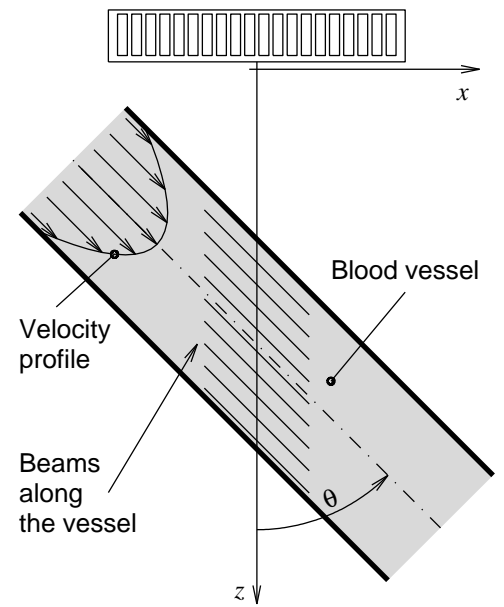

Fig. 12. The directional signals shown inside the vessel are focused along the flow vector direction (from [31]).

beamforming in multiple directions and then find the angle with the highest cross-correlation value [34], [35]:

$$
\begin{aligned}
R_{12 n}\left(\theta_{m}\right) & =\left(\frac{\max \left(R_{12}\left(l, \theta_{m}\right)\right)}{R_{11}\left(0, \theta_{m}\right)}\right) \\
\theta & =\underset{\theta_{m}}{\operatorname{Arg} \max R_{12 n}\left(\theta_{m}\right)}
\end{aligned}
$$

where $\max \left(R_{12}\left(l, \theta_{m}\right)\right)$ is the maximum value of the crosscorrelation function for a given angle and $R_{11}\left(0, \theta_{m}\right)$ is the corresponding power of the signal. The correct angle $\theta$ is found where the normalized correlation function as a function of angle $R_{12 n}\left(\theta_{m}\right)$ has its peak value. For other angles the signals will decorrelate as the scatterers move at different velocities across the beamformed lines. An example of the angle determination is shown in Fig. 13 for synthetic aperture flow. One drawback of the method is, however, that it is very computer intensive.

\section{B. Performance}

An example of SA vector velocity imaging is shown in Fig. 14. A 128 elements linear array transducer was used. The sequence contained 8 emission centers using 17 elements each and a chirp signal for excitation. Data were sampled for 64 


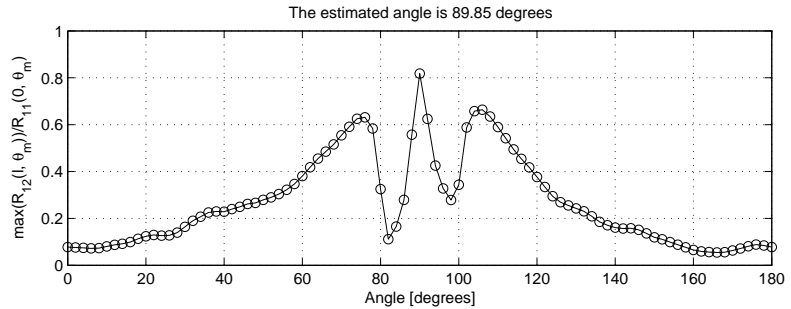

Fig. 13. Normalized maximum correlation as a function of angle. The peak position indicates the correct angle (from [36]).

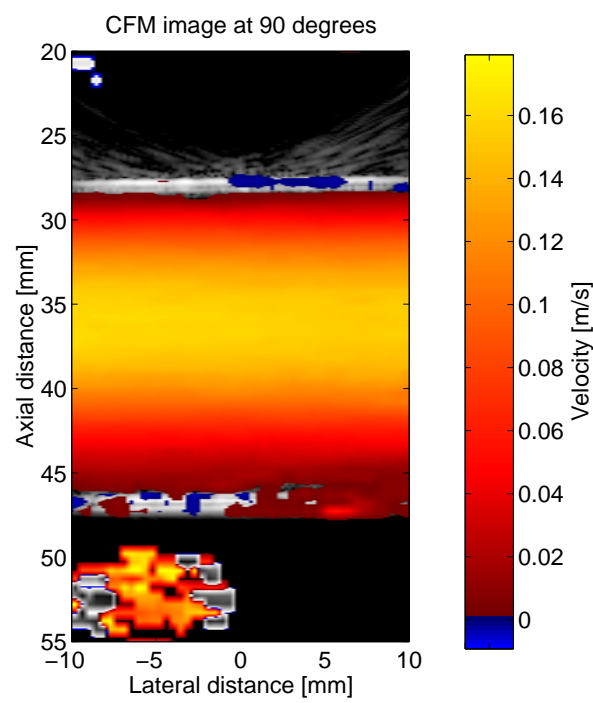

Fig. 14. Synthetic aperture color flow map image of flow rig data at a 90 degree flow angle obtained using 128 emissions (from [31]).

elements at a time centered around the emission elements. The sequence was repeated 16 times for a total of 128 emissions. This makes it possible to make roughly 50 flow images a second to a depth of $15 \mathrm{~cm}$. The flow measured is in a rig with parabolic flow and a flow angle of $90^{\circ}$. The result is shown in Fig. 14. No post processing has been performed on the data. The relative standard deviation is $1.2 \%$ at $90^{\circ}$ and $0.36 \%$ at $60^{\circ}$ [31], and it, thus, possible to attain highly precise quantitative flow measurements with a high temporal resolution.

The angle can also be estimated from the data. The results are shown in Fig. 15 for both $90^{\circ}$ (top) and $60^{\circ}$ (bottom). For $60^{\circ}$ the mean value of all estimates is $60.54^{\circ}$ and the standard deviation is $2.1^{\circ}$. For $90^{\circ}$ the mean value is $90.0003^{\circ}$ and the standard deviation is $1.32^{\circ}$.

\section{Clinical Examples}

An example of an in-vivo SA flow image is shown in Fig. 16. An 8 emission sequence is used with 17 elements combined in transmit and a $20 \mu$ s chirp excitation. Sixteen sets of emissions are used for generating the image [31]. The direction along the flow is encoded as either blue (jugular vein, top) or orange (carotid artery, bottom). No post processing has been performed on the data and raw velocity estimates are displayed. Part of the velocity estimates are missing at the top of the image due to the employment of a chirp.
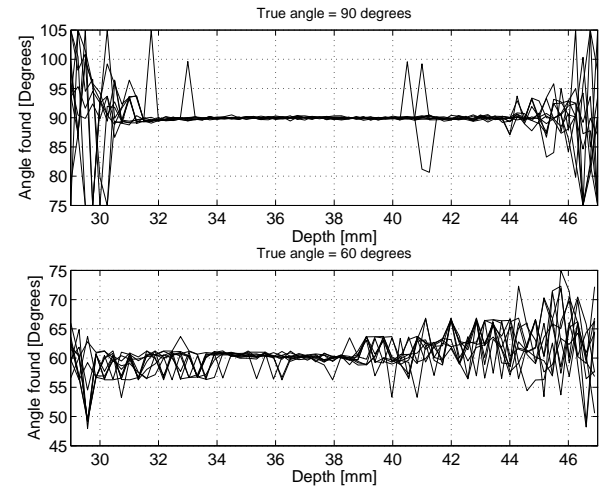

Fig. 15. Estimated velocity angles for a true velocity angle of $90^{\circ}$ (top) and $60^{\circ}$ (from [34])

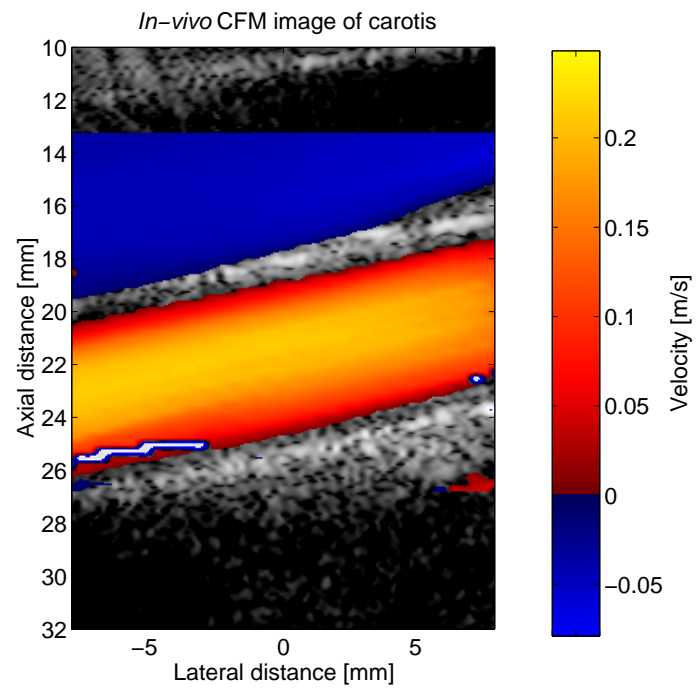

Fig. 16. In-vivo CFM image from the carotid artery using SA flow imaging and directional beamforming (from [31]).

The second clinical example shown in Fig. 17 from the carotid artery also include automatic angle determination by the approach described in [34]. The data is taken at diastole as indicated in the lower graph, where the peak velocity at the center of the vessel is shown.

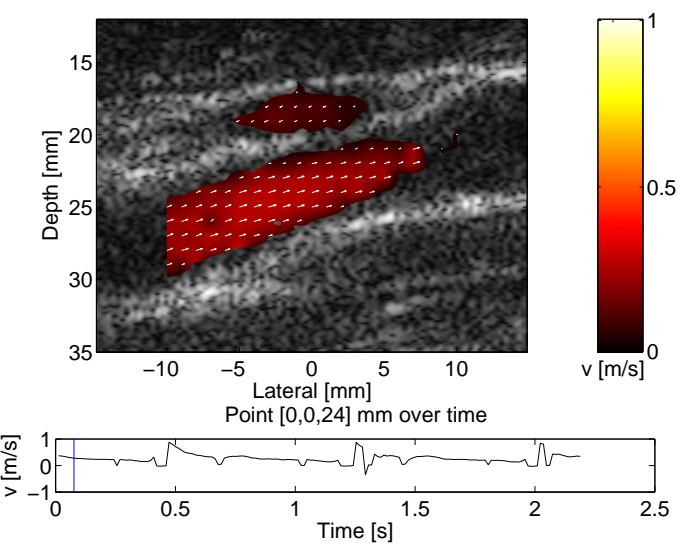

Fig. 17. CFM SA imaging with automatic angle determination (from [34]). 


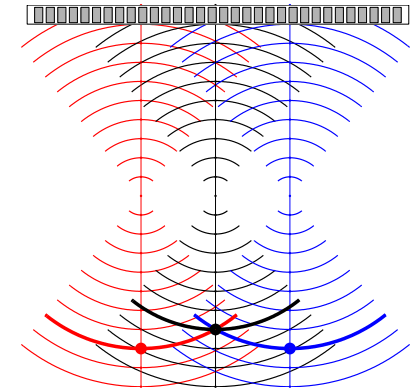

Fig. 18. Fixed focused beams used for dual stage beamforming (from [37]).

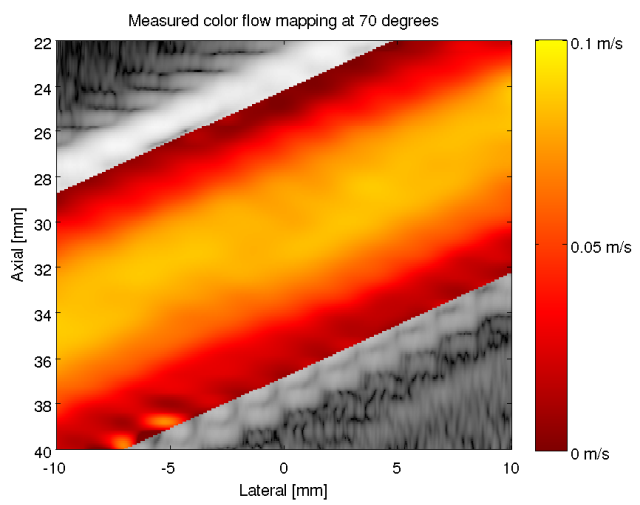

Fig. 19. CFM image generated using dual stage beamforming (from [39]).

\section{Implementation Issues}

A major drawback of SA flow imaging is the large amount of calculations to perform as a full image has to beamformed for each emission and for all received elements. There can be two orders of magnitude more calculations to perform than in a traditional scanner. Methods for reducing the amount are, thus, needed. One possible solution is to use dual stage beamforming [37], [38]. Here a first stage beamformer with a fixed focus in transmit and receive combines the signals from the different elements to a single RF output signal for each emission as shown in Fig. 18. These signals are then combined in a second stage beamformer with dynamic focusing and apodization to yield the synthetic aperture image. This results in two orders of magnitude reduction in the calculational demands and still maintains a good image quality [38].

The approach has been combined with the directional SA beamforming to implement vector flow imaging with dual stage beamforming. An example is shown in Fig. 19, where a color flow map image is generated using 4 fixed focused beams repeated 12 times. The flow angle is $70^{\circ}$ and the parabolic flow of the profile is seen.

\section{Fast Plane Wave Vector Flow}

Another method for acquiring high-frame rate data is to emit plane waves and acquire the signals on all the receive channels. This has been suggested by a number of authors [40], [41], [42]. The advantage is here again that a full image is acquired in very few or one emissions and a very high frame rate can be maintained. This can be combined with the different vector velocity estimation methods and the estimates can be averaged over a long time interval.

Udesen et al. [42], [43], [44] implemented this using a single plane wave emission and speckle tracking for finding the vector velocity. A small reference frame was used for a search in the image to determine both velocity magnitude and direction. A frame rate of $100 \mathrm{~Hz}$ was obtained and several clinical examples were acquired.

\section{A. Clinical Examples}

Fig. 20 shows an example of plane wave vector flow imaging of the carotid artery and jugular vein. The vein is in the top of the images and the carotid artery is seen in a crosssectional view. The jugular vein contains an incompetent valve and during part of the cardiac cycle a reverse flow through the valve is seen in the left most image. Around and after peak systole the flow velocity increase through the valve. Note also the vorticies that appear behind the valve and how they change rotation direction depending on the flow direction. The crosssectional view of the carotid artery also reveals a secondary rotation in the vessel during systole.

\section{Three-Dimensional Vector Flow Imaging}

The vector velocity image in Figs. 4 and 20 shows that the flow in the carotid artery undergoes both a spiral motion after peak systole in the carotid bulb and a helix motion as seen in the cross-sectional image. Such complex motion is found in nearly all other complex vessel geometries, around bifurcation and in both incompetent and functioning valves. There is, thus, a real need for finding the full velocity vector and it is natural to extend the $2 \mathrm{D}$ techniques to $3 \mathrm{D}$.

One possible method is to extend the TO method to $3 \mathrm{D}$ be using a 2D matrix probe [45]. A normal focused field is transmitted and five beams are focused in parallel in receive. One beam for traditional axial velocity estimation, one set of in-phase and quadrature beams in the transverse plane, and on set of in-phase and quadrature beams in the elevation plane are made. The three velocity components can then be estimated independently and at the same time to give the correct 3D vector.

Results from such an approach is shown in Fig. 21, where simulated results from a plug flow is shown. A $32 \times 32$ or 64 $\mathrm{x} 64$ element transducer is used and the velocities in the $x-y$ plane orthogonal to the ultrasound beam direction is estimated. 1024 active elements are used in both cases during transmit and receive. The results indicate that the approach can be used for $3 \mathrm{D}$ vector flow estimation. The arrows indicate the true direction and magnitude and the dashed line is the estimated mean. The circles show one standard deviation.

\section{IMPLiCATIONS AND FUtURE OF Vector Flow IMAGING}

The flow in the human body is pulsating, complex, and hardly ever laminar. The velocity vector changes over time and space, and current static angle compensation methods are inadequate for quantitative velocity estimation in everything 

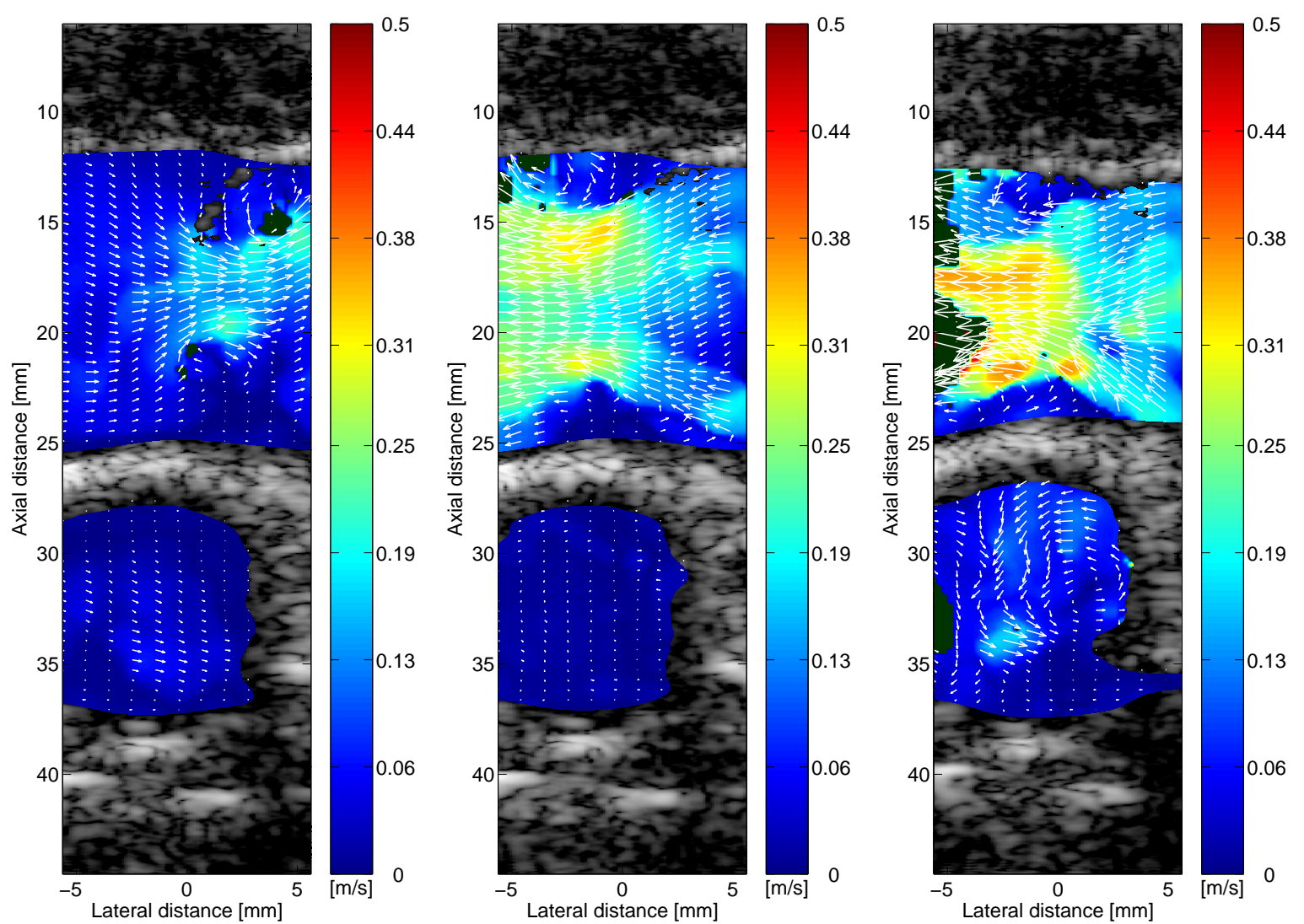

Fig. 20. The images show plane wave vector velocities in the jugular vein (top) and the carotid artery at three different times in the cardiac cycle (from [44]).
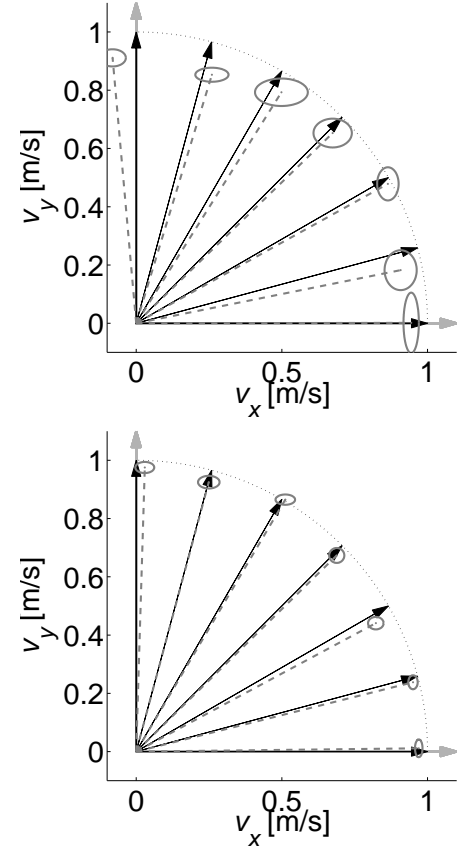

Fig. 21. Three-dimensional vector velocity estimation in the $x-y$ plane orthogonal to the ultrasound beam direction. The top graph shows the estimates for a $32 \times 32$ array and the bottom for a 64 × 64 array (from [45]). but the simplest cases. The new vector velocity imaging schemes are capable of revealing a wealth of information regarding the human circulation in real time and without the use of contrast agents. There is no need for angle correction and the correct magnitude and direction are shown. The early clinical images have visualized the complex flow patterns at bifurcations and valves. The fast vector flow imaging using either synthetic aperture or plane waves demonstrates the fast transitory vorticies and helical flow patterns arising in the arteries and how quickly they change in the cardiac cycle. The secondary flow patterns also demonstrate the need for a full estimation of the three-dimensional velocity vector to fully reveal the flow patterns. Here it is also vital to estimate all three velocity components simultaneously, and this is a main topic for future research.

The vector flow images will reveal a wealth of information and also new quantities can be derived from this data. Ohtsuki and Tanaka [46] showed that the pressure gradients can be calculated from the vector flow, and this will also be one of the future applications of these imaging techniques.

\section{ACKNOWLEDGEMENT}

This work was supported by grant 9700883,9700563 and 26-04-0024 from the Danish Science Foundation and by B-K Medical Aps. This project is also supported by grant 0242008-3 from the Danish Advanced Technology Foundation 


\section{REFERENCES}

[1] D. H. Evans, W. N. McDicken, R. Skidmore, and J. P. Woodcock, Doppler Ultrasound, Physics, Instrumentation, and Clinical Applications. New York: John Wiley \& Sons, 1989.

[2] J. A. Jensen, Estimation of Blood Velocities Using Ultrasound: A Signal Processing Approach. New York: Cambridge University Press, 1996.

[3] D. H. Evans and W. N. McDicken, Doppler Ultrasound, Physics, Instrumentation, and signal processing. New York: John Wiley \& Sons, 2000.

[4] P. J. Kilner, G. Z. Yang, R. H. Mohiaddin, D. N. Firmin, and D. B.Longmore, "Helical and retrograde secondary flow patterns in the aortic arch studied by three-directional magnetic resonance velocity mapping," Circulation, vol. 88, no. 5, pp. 2235 - 2247, 1993.

[5] J. D. Thomas, "Flow in the descending aorta. a turn of the screw or a sideways glance?" Circulation, vol. 82, no. 6, pp. 2263 - 2265, 1990.

[6] D. J. Phillips, K. W. Beach, J. Primozich, and D. E. Strandness, "Should results of ultrasound Doppler studies be reported in units of frequency or velocity?" Ultrasound Med. Biol., vol. 15, pp. 205-212, 1989.

[7] M. D. Fox, "Multiple crossed-beam ultrasound Doppler velocimetry," IEEE Trans. Son. Ultrason., vol. SU-25, pp. 281-286, 1978.

[8] G. E. Trahey, J. W. Allison, and O. T. von Ramm, "Angle independent ultrasonic detection of blood flow," IEEE Trans. Biomed. Eng., vol. BME-34, pp. 965-967, 1987.

[9] H. B. Kim, J. R. Hertzberg, and R. Shandas, "Development and validation of echo PIV," Exp. in Fluids, vol. 36, pp. 455-462, 2004.

[10] L. Liu, H. Zheng, L. Williams, F. Zhang, R. Wang, J. Hertzberg, and R. Shandas, "Development of a custom-designed echo particle image velocimetry system for multi-component hemodynamic measurements: system characterization and initial experimental results," Phys. Med. Biol., vol. 53, pp. 1397-1412, 2008.

[11] V. L. Newhouse, D. Censor, T. Vontz, J. A. Cisneros, and B. B. Goldberg, "Ultrasound Doppler probing of flows transverse with respect to beam axis," IEEE Trans. Biomed. Eng., vol. BME-34, pp. 779-788, 1987.

[12] P. Tortoli, G. Guidi, F. Guidi, and C. Atzeni, "A review of experimental transverse Doppler studies," IEEE Trans. Ultrason., Ferroelec., Freq. Contr., vol. 41, pp. 84-89, 1994b.

[13] P. Tortoli, G. Bambi, and S. Ricci, "Accurate doppler angle estimation for vector flow measurements," IEEE Trans. Ultrason., Ferroelec., Freq. Contr., vol. 53, no. 8, pp. 1425 - 1431, Aug. 2006.

[14] B. Dunmire and K. W. Beach, "A Brief History of Vector Doppler," in SPIE Med. Imag. V Symp., vol. 4325, Feb. 2001, pp. 200-214.

[15] D. H. Evans, J. A. Jensen, and M. B. Nielsen, "Ultrasonic colour Doppler imaging," Interface Focus, vol. 1, no. 4, pp. 490-502, August 2011.

[16] J. A. Jensen and P. Munk, "A New Method for Estimation of Velocity Vectors," IEEE Trans. Ultrason., Ferroelec., Freq. Contr., vol. 45, pp. 837-851, 1998.

[17] M. E. Anderson, "Multi-dimensional velocity estimation with ultrasound using spatial quadrature," IEEE Trans. Ultrason., Ferroelec., Freq. Contr., vol. 45, pp. 852-861, 1998.

[18] J. A. Jensen and N. B. Svendsen, "Calculation of Pressure Fields from Arbitrarily Shaped, Apodized, and Excited Ultrasound Transducers," IEEE Trans. Ultrason., Ferroelec., Freq. Contr., vol. 39, pp. 262-267, 1992.

[19] J. A. Jensen, "Field: A Program for Simulating Ultrasound Systems," Med. Biol. Eng. Comp., vol. 10th Nordic-Baltic Conference on Biomedical Imaging, Vol. 4, Supplement 1, Part 1, pp. 351-353, 1996.

[20] J. Udesen and J. A. Jensen, "Investigation of Transverse Oscillation Method," IEEE Trans. Ultrason., Ferroelec., Freq. Contr., vol. 53, pp. 959-971, 2006.

[21] P. Munk and J. A. Jensen, "Performance of velocity vector estimation using an improved dynamic beamforming setup," in Proc. of SPIE: Progress in biomedical optics and imaging, vol. 4325, 2001, pp. 227241.

[22] J. A. Jensen, "A New Estimator for Vector Velocity Estimation," IEEE Trans. Ultrason., Ferroelec., Freq. Contr., vol. 48, no. 4, pp. 886-894, 2001.

[23] J. A. Jensen, O. Holm, L. J. Jensen, H. Bendsen, S. I. Nikolov, B. G. Tomov, P. Munk, M. Hansen, K. Salomonsen, J. Hansen, K. Gormsen, H. M. Pedersen, and K. L. Gammelmark, "Ultrasound Research Scanner for Real-time Synthetic Aperture Image Acquisition," IEEE Trans. Ultrason., Ferroelec., Freq. Contr., vol. 52 (5), pp. 881-891, May 2005.
[24] J. Udesen, M. B. Nielsen, K. R. Nielsen, and J. A. Jensen, "Examples of in-vivo blood vector velocity estimation," Ultrasound Med. Biol., vol. 33, pp. 541-548, 2007.

[25] K. L. Hansen, J. Udesen, C. Thomsen, J. A. Jensen, and M. B. Nielsen, "In-vivo validation of transverse oscillation vector velocity estimation with MR angiography," IEEE Trans. Ultrason., Ferroelec., Freq. Contr., vol. 56, no. 1, pp. 91-100, 2009.

[26] P. M. Hansen, M. M. Pedersen, K. L. Hansen, M. B. Nielsen, and J. A. Jensen, "Examples of vector velocity imaging," in 15. Nordic-Baltic Conf. Biomed. Eng. and Med. Phys., 2011.

[27] J. A. Jensen, S. Nikolov, K. L. Gammelmark, and M. H. Pedersen, "Synthetic aperture ultrasound imaging," Ultrasonics, vol. 44, pp. e5e15, 2006.

[28] K. L. Gammelmark and J. A. Jensen, "Multielement synthetic transmit aperture imaging using temporal encoding," IEEE Trans. Med. Imag., vol. 22, no. 4, pp. 552-563, 2003.

[29] M. H. Pedersen, T. X. Misaridis, and J. A. Jensen, "Clinical evaluation of chirp-coded excitation in medical ultrasound," Ultrasound Med. Biol., vol. 29, no. 6, pp. 895-905, 2003.

[30] S. I. Nikolov and J. A. Jensen, "Velocity estimation using synthetic aperture imaging," in Proc. IEEE Ultrason. Symp., 2001, pp. 1409-1412.

[31] J. A. Jensen and S. I. Nikolov, "Directional synthetic aperture flow imaging," IEEE Trans. Ultrason., Ferroelec., Freq. Contr., vol. 51, pp. $1107-1118,2004$

[32] J. A. Jensen, "Directional velocity estimation using focusing along the flow direction: I: Theory and simulation," IEEE Trans. Ultrason., Ferroelec., Freq. Contr., vol. 50, pp. 857-872, 2003.

[33] J. A. Jensen and R. Bjerngaard, "Directional velocity estimation using focusing along the flow direction: II: Experimental investigation," IEEE Trans. Ultrason., Ferroelec., Freq. Contr., vol. 50, pp. 873-880, 2003.

[34] J. A. Jensen and N. Oddershede, "Estimation of velocity vectors in synthetic aperture ultrasound imaging." IEEE Trans. Ultrason., Ferroelec., Freq. Contr., vol. 25, pp. 1637-1644, 2006.

[35] J. Kortbek and J. A. Jensen, "Estimation of velocity vector angles using the directional cross-correlation method," IEEE Trans. Ultrason., Ferroelec., Freq. Contr., vol. 53, pp. 2036-2049, 2006.

[36] J. A. Jensen, "Velocity vector estimation in synthetic aperture flow and B-mode imaging," in IEEE International Symposium on Biomedical imaging from nano to macro, 2004, pp. 32-35.

[37] J. Kortbek, J. A. Jensen, and K. L. Gammelmark, "Synthetic aperture sequential beamforming," in Proc. IEEE Ultrason. Symp., 2008, pp. $966-$ 969.

[38] M. C. Hemmsen, P. M. Hansen, T. Lange, J. M. Hansen, M. B. Nielsen, and J. A. Jensen, "Preliminary In-Vivo evaluation of Synthetic Aperture Sequential Beamformation using a multielement convex array," in Proc. IEEE Ultrason. Symp., Oct. 2011, p. Submitted.

[39] Y. Li and J. A. Jensen, "Directional synthetic aperture flow imaging using a dual stage beamformer approach," in Proc. IEEE Ultrason. Symp., 2011, p. Accepted.

[40] J. Bercoff, M. Tanter, L. Sandrin, S. Catheline, and M. Fink, "Ultrafast compound imaging for 2-d displacment vector measurements: application to transient elastography and color flow mapping," in Proc. IEEE Ultrason. Symp., 2001, pp. 1619-1622.

[41] M. Tanter, J. Bercoff, L. Sandrin, and M. Fink, "Ultrafast Compound Imaging for 2-d Motion Vector Estimation: application to Transient Elastography," IEEE Trans. Ultrason., Ferroelec., Freq. Contr., vol. 49, pp. 1363-1374, 2002 .

[42] J. Udesen, F. Gran, and J. A. Jensen, "Fast Color Flow Mode Imaging Using Plane Wave Excitation and Temporal Encoding," in Proc. SPIE - Progress in biomedical optics and imaging, vol. 5750, Feb. 2005, pp. 427-436.

[43] J. Udesen, F. Gran, K. L. Hansen, J. A. Jensen, C. Thomsen, and M. B. Nielsen, "High frame-rate blood vector velocity imaging using plane waves: Simulations and preliminary experiments," IEEE Trans. Ultrason., Ferroelec., Freq. Contr., vol. 55, no. 8, pp. 1729-1743, 2008.

[44] K. L. Hansen, J. Udesen, F. Gran, J. A. Jensen, and M. B. Nielsen, "In-vivo examples of complex flow patterns with a fast vector velocity method," Ultraschall in Med, vol. 30, pp. 471-476, 2009.

[45] M. J. Pihl and J. A. Jensen, "3D velocity estimation using a 2D phased array," in Proc. IEEE Ultrason. Symp., 2011, p. Accepted.

[46] S. Ohtsuki and M. Tanaka, "Doppler pressure field deduced from the Doppler velocity field in an observation plane in a fluid," Ultrasound Med. Biol., vol. 29, no. 10, pp. 1431-1438, 2003. 